\title{
METABOLIC ENGINEERING OF MEP PATHWAY FOR OVERPRODUCTION OF LYCOPENE IN SACCHAROMYCES CEREVISIAE USING PESC-LEU AND PTEF1/ZEO VECTORS
}

\author{
AlsheHri, W. A. ${ }^{1}$ - GADALLA, N. O. ${ }^{2,3}$-EDRIS, S. ${ }^{4,5,6}$ - ATEF, A. ${ }^{4}$ - HEMEG, H. ${ }^{7}$ - AL-QUWAIE, \\ D. A. $\mathrm{H}^{8}{ }^{8}$ - AlsubHI, N. H. ${ }^{8}-$ KABLI, S. A. ${ }^{4}-$ BAHIELDIN, A. ${ }^{4,6^{*}}$ \\ ${ }^{I}$ Department of Biology, College of Science, University of Jeddah, Jeddah, Saudi Arabia \\ ${ }^{2}$ Department of Arid Land Agriculture, Faculty of Meteorology, Environment and Arid Land \\ Agriculture, King Abdulaziz University, Jeddah, Saudi Arabia \\ ${ }^{3}$ Genetics and Cytology Department, Genetic Engineering and Biotechnology Division, \\ National Research Center, Dokki, Egypt \\ ${ }^{4}$ Department of Biological Sciences, Faculty of Science, King Abdulaziz University, Jeddah, \\ Saudi Arabia \\ ${ }^{5}$ Princess Al Jawhara Albrahim Centre of Excellence in Research of Hereditary Disorders \\ (PACER-HD), King Abdulaziz University, Jeddah, Saudi Arabia \\ ${ }^{6}$ Department of Genetics, Faculty of Agriculture, Ain Shams University, Cairo, Egypt \\ ${ }^{7}$ Department of Medical Laboratory Technology, Faculty of Applied Sciences, Tiba University, \\ Al-Madina Al-Monawara, Saudi Arabia \\ ${ }^{8}$ Department of Biological Sciences, Rabigh College of Science and Arts, King Abdulaziz \\ University (KAU), Rabigh, Saudi Arabia \\ *Corresponding author \\ e-mail: abmahmed@kau.edu.sa; phone: +966-506-329-922
}

(Received $29^{\text {th }}$ Jan 2020; accepted $2^{\text {nd }}$ Jul 2020)

\begin{abstract}
Recent studies indicated that overexpression of $d x s$ and $i d i$ genes in bacteria transformed with crtEBI genes dramatically improved the production of lycopene. The aim of the present study was the assessment of $c r t E B I$-transformed S. cerevisiae overexpressing the bacterial synthetic $d x s$ and yeast $i d i$ genes for lycopene production. The two genes were driven by galactose-induced promoters namely GALI and GAL10, respectively, of the pESC-LEU yeast vector of which gene cassettes were inserted in pTEF1/Zeo vector. The synthetic $d x s$ gene was constructed in accordance with the preferred codon usage in S. cerevisiae with no change in the resulting amino acid sequences. The RT-PCR analysis indicated that the two genes were efficiently transcribed in crtEBI-transformed $S$. cerevisiae cells. The highest production of lycopene ( $6755 \mu \mathrm{g}$ lycopene/g dry cell weight) was reached for yeast cells transformed with the two genes, which is higher than the previously reported lycopene levels in yeast. The level of AcetylCoenzyme A (Acetyl-CoA) was negatively related to that of lycopene in transformed S. cerevisiae cells, suggesting that lycopene and Acetyl-CoA biosyntheses compete for the use of pyruvate.
\end{abstract}

Keywords: GAL1 promoter, GAL10 promoter, RT-PCR, zeocin resistance, Acetyl-CoA, pESC-LEU yeast vector, pTEF1/Zeo vector

Abbreviations: IPP, isopentenyl pyrophosphate; DMAPP, dimethylallyl diphosphate; DXP, 1-deoxy-Dxylulose 5-phosphate; DXS, 1-deoxy-D-xylulose 5-phosphate synthase; GAP, glyceraldehyde-3phosphate; IPDP, isopentenyl diphosphate; FPP, farnesyl diphosphate; DCW, dry cell weight; TCA, tricarboxylic acid; MDH, malate dehydrogenase; ANOVA, analysis of variance; LSD, least significant differences 


\section{Introduction}

Lycopene is an important naturally occurring intermediate metabolite directly converted into $\beta$-carotene. Lycopene received major interest among carotenoids as it may be beneficial in diseases such as cancer, coronary heart disease and many other chronic conditions including osteoporosis. It is also used as an antioxidant to reduce cellular or tissue damage (Sevgili and Erkman, 2019). Metabolic engineering was successfully employed in improving industrial strains (Lee et al., 2005; Price et al., 2004) through the overproduction of a desired product via expression/overexpression of certain metabolic genes (Herrgård et al., 2006; Kirschner, 2005). Isoprenoids, basically formed from two precursors, isopentenyl pyrophosphate (IPP) and its isomer dimethylallyl diphosphate (DMAPP), have many applications in biotechnology and industry (Lange and Croteau, 1999; Römer et al., 2000). Biosynthesis of these two precursors utilizes the 2-C-methyl-D-erythritol 4-phosphate (MEP) pathway in eukaryotes and eubacteria (Eisenreich et al., 2004). This pathway supports the synthesis of the major pigments, including lycopene, hormones and mono- and di-terpenes (Eisenreich et al., 2004) by initiation of a reaction catalyzed by the enzyme 1-deoxy-Dxylulose 5-phosphate (DXP) synthase (DXS) using pyruvate and glyceraldehyde-3phosphate (GAP) as substrates (Fig. 1a) to yield DXP (Lange et al., 1998; Lois et al., 1998). The latter is converted into a mixture of IPP and DMAPP compounds through six consecutive enzymatic reactions (Lange et al., 2000; Rodriguez-Concepción and Boronat, 2002). The fluctuation in ratio of these two compounds is based on the expression of a gene encoding isopentenyl diphosphate (IPDP) isomerase (the idi gene), a key enzyme that has been shown to exert a positive effect on lycopene production (Lee and Schmidt-Dannert, 2002). Recent studies indicated that overexpression of $d x s$ and $i d i$ genes in E. coli transformed with crtEBI genes resulted in the production of lycopene at $8.57 \mathrm{mg} / \mathrm{g}$ dry cell weight (DCW), which is $\sim 6$-fold higher than the control crtEBI-E. coli strain (1.48 mg/g DCW) (Choi et al., 2010). On the other hand, the overexpression of the $m d h$ gene, which encodes malate dehydrogenase (MDH) of the tricarboxylic acid (TCA) cycle, in conjunction with $i d i$ and $d x s$ genes, resulted in a slightly higher level of lycopene $(9.98 \mathrm{mg} / \mathrm{g}$ DCW) (Choi et al., 2010). TCA cycle has no direct connection with MEP pathway for lycopene production, except that NADH generated by MDH action is required for the synthesis of isopentenyl diphosphate (IPP) or dimethylallyl diphosphate (DMAPP) via the MEP pathway. In cilico analysis for improving lycopene production in E. coli (Choi et al., 2010) indicated that the flux from pyruvate to Acetyl-CoA (Fig. 1b) is decreased and flux from pyruvate to DXP is increased due to overexpression of $d x s$ and $i d i$ genes. This indicates that the MEP pathway (for lycopene biosynthesis) and Acetyl-CoA biosynthesis (precursor for the TCA cycle, glyoxylate cycle and histone acetylation) might compete for the use of pyruvate in E. coli (Fig. 1).

More recently, Sun et al. (2014) has utilized a completely different approach to accumulate higher levels of lycopene $(6.5 \mathrm{mg} / \mathrm{g} \mathrm{DCW})$ in engineered E. coli. This approach relies on the deletion of genes (e.g., crtY and $\operatorname{crt} X$ ) functioning downstream the final step of lycopene biosynthesis. The authors also used ribosome binding site libraries to modulate expression of $d x s$, idi and the crt genes and optimal strain produced as high as $50.6 \mathrm{mg} / \mathrm{g}$ DCW. More recently, the overexpression of OLE1 gene, encoding stearoyl-CoA 9-desaturase, in combination with the STB5 gene, encoding a transcription factor involved in NADPH production, in the yeast with increasing membrane flexibility and NAPDH production resulted in the production of up to 
$41.8 \mathrm{mg} / \mathrm{g}$ DCW of lycopene (Hong et al., 2019). These two genes can be further used in addition to the genes under study in order to maximize the production of lycopene in yeast (S. cerevisiae).

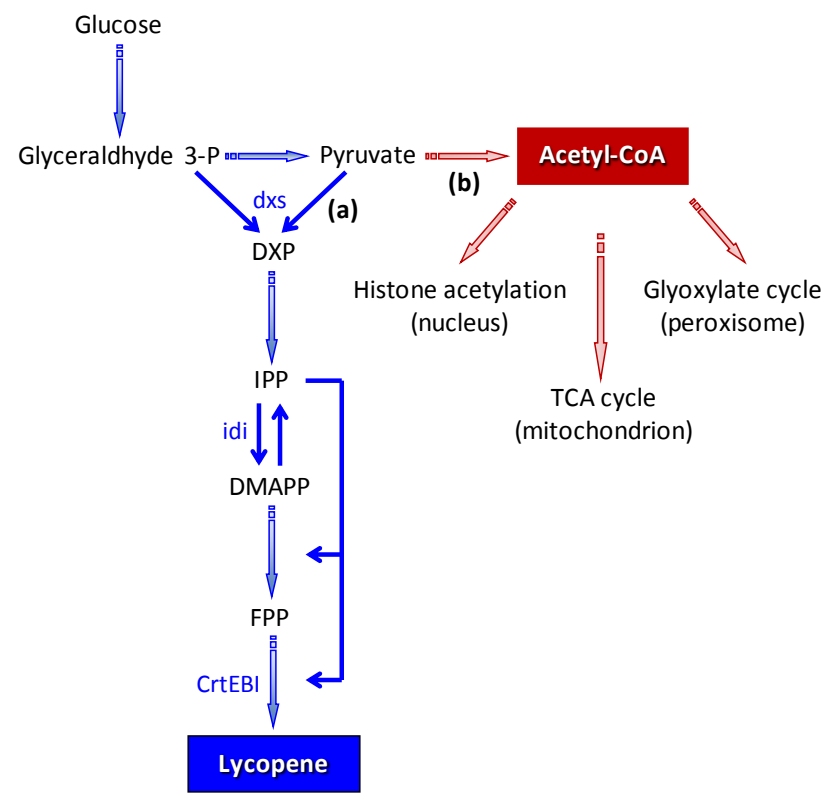

Figure 1. Engineered MEP pathway (in blue) for lycopene biosynthesis (a) by inducing the conversion of pyruvate to DXP due to the action of the bacterial dxs and overexpressing the yeast idi gene in crtEBI-transformed S. cerevisiae cells. Acetyl-CoA biosynthesis $(b)$ and downstream metabolic processes (in red) in the nucleus (histone acetylation), mitochondrion (TCA cycle) and peroxisome (glyoxylate cycle) are favored in nature due to the conversion of pyruvate to Acetyl-CoA. Abbreviations: DXP, 1-deoxy-D-xylulose-5-phosphate; IPP, isopentenyl pyrophosphate; DMAPP, dimethylallyl diphosphate; FPP, farnesyl diphosphate

The aim of the present study was the assessment of crtEBI-transformed $S$. cerevisiae line overexpressing the bacterial $d x s$ and the yeast $i d i$ genes for lycopene production. The sequence of the bacterial $d x s$ gene was optimized according to the preferred codon usage of $S$. cerevisiae. The two genes were efficiently expressed under the control of GAL1 and GAL10 promoters, respectively, contained within the pESC-LEU vector (cat. no. 217452, Agilent Technologies, Santa Clara, CA 95051, USA).

\section{Materials and methods}

\section{Strain and its growth conditions}

Master plates and cell cultures of Saccharomyces cerevisiae DSY-5 strain (Dualsystems Biotech AG, Schlieren, Switzerland) were prepared as indicated earlier (Bahieldin et al., 2014).

\section{Construction of yeast expression vector with the synthetic bacterial dxs and the native yeast idi genes}

The synthetic $d x s$ gene (1863 bp) was constructed by Bioneer corporation (Daejeon, Republic of Korea; NCBI submission ID no. 1719788) and inserted in the pKS1 
(6.65 bp) shuttle vector (cat. no. P03305, KickStart ${ }^{\mathrm{TM}}$ Dualsystems Biotech AG, Schlieren, Switzerland). The gene was synthesized in reference to the corresponding native gene from E. coli, strain K-12 (acc. no. HG738867, region: 340298-342160 bp) and the codon preference of $S$. cerevisiae. The replacement of nucleotides between the native and synthetic gene resulted in the reduction of GC contents from $\sim 56 \%$ to $\sim 46 \%$. Added-on restriction sites of BamHI and KpnI were inserted at the $5{ }^{`}$ and $3^{\prime}$ ends of the synthesized gene, respectively, to facilitate further ligation of the gene to the BamHI/KpnI-digested pESC-LEU vector $(7.8 \mathrm{~kb})$ downstream of the GAL1 promoter (Fig. 2). The yeast idi full-length open reading frame (acc. no. NM_001183931, 867 bp) was amplified from $S$. cerevisiae DSY-5 strain by PCR using primers (idi_F: 5 attagaattc-ATGACTGCCGACAACAATAGTA $3{ }^{\prime}$ and idi_R: 5 attagagctcTTATAGCATTCTATGAATTTGCCTGTC 3') with EcoRI and SacI restriction sites added-on at the $5^{\prime}$ and $3^{\prime}$ ends, respectively. Primers were designed using the Universal ProbeLibrary Assay Design Center (Roche, www.roche-appliedscience.com, 2012). PCR was performed in a $20-\mu 1$ reaction mixture and conditions consisted of denaturation at $94^{\circ} \mathrm{C}$ for $4 \mathrm{~min}$ (first cycle), then denaturation for $15 \mathrm{~s}$, annealing at $48{ }^{\circ} \mathrm{C}$ for $30 \mathrm{~s}$, and extension at $72^{\circ} \mathrm{C}$ for $1 \mathrm{~min}$ (40 cycles). Amplicons were run on a $1.2 \%$ agarose gel stained with ethidium bromide and visualized using the Gel Doc XR (Bio-Rad Laboratories; Hercules, CA, USA). EcoRI/SacI-digested PCR products were ligated to EcoRI/SacI-digested pESC-LEU vector downstream of the GAL10 promoter (Fig. 2). Generated P-GAL1/dxs/T-CYC and P-GAL1/dxs/T-CYC::PGAL10/idi/T-ADH1 cassettes were digested with ApaI/NotI and ligated to ApaI/NotIdigested pTEF1/Zeo vector (3.56 kb, cat. no. V503-20, Life Technologies Inc., Grand Island, NY, USA) to generate $\mathrm{pTEF} 1 / \mathrm{Zeo} / d x s$ and $\mathrm{pTEF} 1 / \mathrm{Zeo} / d x s / i d i$ yeast expression vectors.

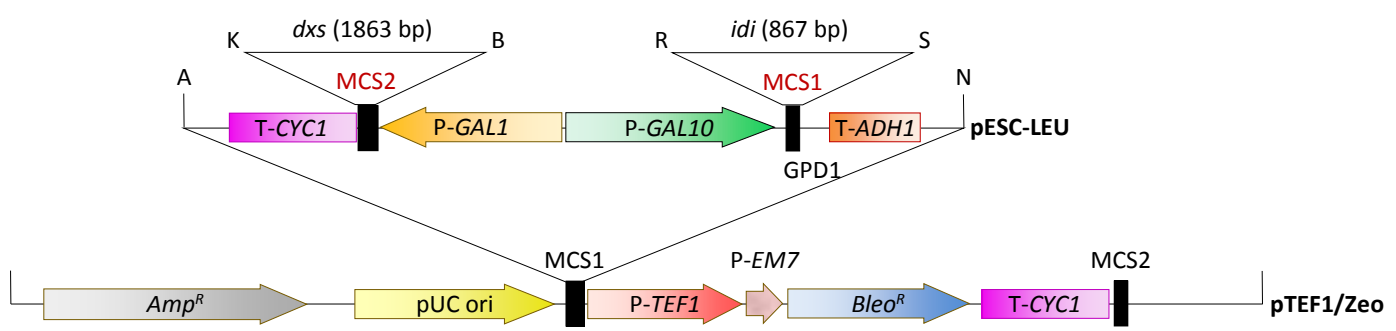

Figure 2. Schematic representation of P-GAL1/dxs/T-CYC::P-GAL10/idi/T-ADH1 gene cassette $(\sim 4.23 \mathrm{~kb})$ of yeast expression pESC-LEU vector construct after genes were inserted between $R / S$ and $B / K$ sites of MCS1 and MCS2 (red), respectively. Gene cassette was inserted between N/A sites of MCS1 (black) of yeast expression pTEF1/Zeo $(\sim 3.56 \mathrm{~kb})$ vector construct. Abbreviations: T-CYCl and T-ADH1: yeast CYCl and ADH1 terminators, P-GAL1 and PGAL10: yeast GAL1 and GAL10 divergent promoters, MCS1 and MCS2: multiple cloning sites 1 and 2, Amp ${ }^{R}$ : ampicillin prokaryotic resistance gene, $p U C$ ori: origin of replication, P-TEF1 and P-EM7: TEF and EM7 eukaryotic and prokaryotic promoters, respectively, Bleo ${ }^{R}$ : Zeocin prokaryotic and eukaryotic resistance gene. Abbreviations of restriction enzymes: A: ApaI, K: KpnI, B: BamHI, R: EcoRI, S: SacI, N: NotI

\section{Transformation of $S$. cerevisiae with a vector harboring the idi/dxs genes}

The $S$. cerevisiae cell Line 2 with pKS1 harboring synthetic crtEBI genes (Bahieldin et al., 2014) was transformed with pTEF1/Zeo, pTEF1/Zeo/dxs or $\mathrm{pTEF1/Zeo/dxs/idi}$ yeast expression vector following protocol in the manufacturer's manual (KickStart ${ }^{\mathrm{TM}}$ 
Dualsystem protein expression kit protocol, Schlieren, Switzerland). The S. cerevisiae cell Line 2 as well as those harboring the pTEF1/Zeo derivatives were grown under optimized growth conditions $\left(35^{\circ} \mathrm{C}\right.$ with YPD medium adjusted at $\mathrm{pH} 6.0$ and supplemented with $0.5 \%$ glucose and $80 \mu \mathrm{g} / \mathrm{ml} \mathrm{G} 418$ ) as described earlier (Bahieldin et al., 2014). Yeast cell Line 2 harboring pTEF1/Zeo derivatives were originally selected on $100 \mu \mathrm{g} / \mathrm{ml}$ zeocin (cat. no. R250-01, Life Technologies Inc., Grand Island, NY, USA) with no galactose added to the medium. Transformed cells with robust growth on YPD plates were recovered and stored as glycerol stocks at $-80^{\circ} \mathrm{C}$. Then, the growth behavior in terms of biomass production of the original $S$. cerevisiae DSY-5 transformed cell Line 2 was tested in the presence of zeocin $(0,50,100$ and $200 \mu \mathrm{g} / \mathrm{ml})$ in the medium to detect the concentration completely inhibiting cell growth. This concentration of zeocin was used in evaluating yeast cells transformed with different pTEF1/Zeo derivatives. Biomass was determined under optimized conditions (cells were grown in YPD medium with $0.5 \%$ glucose at $\mathrm{pH} 6.0 / 35^{\circ} \mathrm{C}$, see Bahieldin et al., 2014), in the presence of $200 \mu \mathrm{g} / \mathrm{ml}$ zeocin) in five randomly selected $S$. cerevisiae Line 2 transformed with pTEF1/Zeo, pTEF1/Zeo/dxs or pTEF1/Zeo/dxs/idi expression vector. Turbidity was monitored by spectrophotometer at $546 \mathrm{~nm}$ across growth time $(0$, $5,25,45$ and $65 \mathrm{~h}$ ), in three replicates. The best performing $S$. cerevisiae Line 2 transformed with pTEF1/Zeo, pTEF1/Zeo/dxs and pTEF1/Zeo/dxs/idi expression vectors were used in further experiments.

\section{RT-PCR analysis}

Transcript levels of the $d x s$ and/or $d x s / i d i$ genes in selected single events (based on biomass production) of $S$. cerevisiae cell Line 2 with pTEF1/Zeo/dxs or pTEF1/Zeo/dxs/idi yeast expression vector, grown at optimized conditions in the presence of galactose $(2 \%)$ for $65 \mathrm{~h}$, were analyzed by RT-PCR. Transformed yeast cells with pTEF1/Zeo vector only were used as a control. RNAs were extracted with QIAzol $^{\circledR}$ (Qiagen, Düsseldorf, Germany) from different transformed derivatives of the S. cerevisiae cell Line 2. Then, RNAs were treated with RNase-free DNase (Promega, Madison, WI, USA). First-strand cDNA was synthesized using $1 \mu \mathrm{g}$ of total RNA, $0.5 \mu \mathrm{g}$ oligo reverse primers of each gene (Table 1) and Superscript II reverse transcriptase (Invitrogen, Carlsbad, CA, USA) to a final volume of $20 \mu 1$. PCR with forward and reverse primers (Table 1) for amplifying either gene consisted of denaturation at $94{ }^{\circ} \mathrm{C}$ for $4 \mathrm{~min}$ (first cycle), then denaturation for $15 \mathrm{~s}$, annealing at $48{ }^{\circ} \mathrm{C}$ for $30 \mathrm{~s}$, and extension at $72{ }^{\circ} \mathrm{C}$ for $45 \mathrm{~s}$ (40 cycles) to amplify 294 bp for $d x s$ gene and $288 \mathrm{bp}$ for idi gene. Amplicons were run on a 1.2\% agarose gel stained with ethidium bromide and visualized using the Gel Doc XR (Bio-Rad Laboratories; Hercules, CA, USA). To ensure the absence of DNA contamination, PCR for the original RNA samples was run and results were negative (data not shown). Yeast actin gene (acc. no. L00026.1) was used as a reference to amplify 337 bp (Table 1).

\section{Quantification of lycopene and acetyl-coenzyme $A$ in S. cerevisiae dxs- and dxs/idi- transformed cells}

Quantification of lycopene in S. cerevisiae cell Line 2 as well as in single selected transformed yeast cell with pTEF1/Zeo, pTEF1/Zeo/dxs or pTEF1/Zeo/dxs/idi expression vector was performed as described (Bahieldin et al., 2014). First, cells were harvested by centrifugation and lyophilized. The extracted lycopene was subjected to 
high-performance liquid chromatography (HPLC) as described (Miura et al., 1998a). The $S$. cerevisiae cell Line 2 was used as a control. Galactose was added to the optimized medium at different concentrations $(0,1,2,3$ and 4\%) and measurements were made after $65 \mathrm{~h}$ growth. Concurrently, Acetyl-Coenzyme A Assay kit (cat. no. MAK039, Sigma-Aldrich, St. Louis, MO, USA) was used to detect concentration of Acetyl-CoA in nmole/ $\mu \mathrm{l}$ after $65 \mathrm{~h}$ growth as described in the manufacturer's manual. Deproteinized samples were collected from the different $d x s$ - or $d x s / i d i$-transformed lines as well as from $S$. cerevisiae cell Line 2 (control). Then, time-dependent profiles of lycopene and Acetyl-CoA syntheses in different transformed cells were tested across time $(0,5,25,45$ and $65 \mathrm{~h})$ when cells were grown under optimized growth condition and optimal concentration of galactose. Experiments were statistically analyzed via twoway analysis of variance (ANOVA) with three replicates. ANOVA was performed based on the data of lycopene and Acetyl-CoA levels for the four yeast transformants with different vectors under the five different galactose concentrations. Multiple comparisons were performed within and across these two factors based on the least significant differences (LSD) at 5\%. P-values were estimated for the variances among transformants with different vectors (V) and among different galactose (G) concentrations as well as for the variance due to the interaction between these two factors (e.g., V x G).

Table 1. Primer names, sequences, expected sizes and locations of amplicons in the coding regions of the synthetic dxs of bacterial origin (E. coli, strain $K-12)$ and yeast endogenous idi genes along with the house-keeping gene actin of S. cerevisiae

\begin{tabular}{|c|c|c|c|}
\hline Primer & Accession no. & Sequence $(5-3)$ & Size of amplicon (bp) \\
\hline $\begin{array}{l}d x s_{-} \mathrm{RT} \_\mathrm{F} \\
d x s_{-} \mathrm{RT} \_\mathrm{R}\end{array}$ & HG738867 & $\begin{array}{l}\text { GAA GCT ATG AAC CAT GCA GG } \\
\text { AAC ATC ATG ACC ATC AAC TGG }\end{array}$ & 294 \\
\hline $\begin{array}{l}i d i \_\mathrm{RT} \_\mathrm{F} \\
i d i \_\mathrm{RT} \_\mathrm{R}\end{array}$ & NM_001183931 & $\begin{array}{l}\text { ACG TCA AAT GAC GAA AGC G } \\
\text { ACA TAG TGG ATG AGA GCA GC }\end{array}$ & 288 \\
\hline $\begin{array}{l}\text { actin- }-\mathrm{F} \\
\text { actin- } \mathrm{R}\end{array}$ & L00026.1 & $\begin{array}{l}\text { CCA ATT GCT CGA GAG ATT TC } \\
\text { CAT GAT ACC TTG GTG TCT TG }\end{array}$ & 337 \\
\hline
\end{tabular}

\section{Results}

Yeast strains tend to respond to antibiotics differently. Zeocin antibiotic was tested at different concentrations $(0,50,100$ and $200 \mu \mathrm{g} / \mathrm{ml})$ against the yeast Line 2 transformed with the three $c r t E B I$ genes (Bahieldin et al., 2014) and results indicated that zeocin at $200 \mu \mathrm{g} / \mathrm{ml}$ completely inhibited growth of yeast cells in terms of biomass production. This concentration was used to select one, out of five, single transformed yeast cell - in terms of biomass production - with pTEF1/Zeo vector or that harboring $d x s$ or $d x s / i d i$ genes, both functions upstream the crt genes in the MEP pathway. There is no significant difference in terms of biomass within each of the three transformants (with pTEF1/Zeo or derivatives) after 45 and $65 \mathrm{~h}$ indicating that cell growth almost reached a growth plateau (Fig. 3) in accordance with the findings of Bahieldin et al. (2014), where they found that $45 \mathrm{~h}$ were sufficient to recover the highest biomass production of crtEBI transformants. The crtEBI genes were inserted in the pKS1 vector under the control of $A D H 2$ promoter, while the other two upstream genes were inserted in the pTEF1/Zeo vector under the control of GAL1 promoter for $d x s$ gene and the control of 
GAL10 promoter for idi gene. Expression of the $c r t$ genes requires the depletion of glucose, while expression of the latter two genes requires the presence of galactose. It was decided to use the transformed Line EBI-Nil2 with pTEF1/Zeo vector only (Fig. 3a), the transformed Line EBI-D4 with $d x s$ gene (Fig. 3c) and transformed Line EBI-DI2 with $d x s$ /idi genes (Fig. $3 b$ ) in the presence of $200 \mu \mathrm{g} / \mathrm{ml}$ zeocin in the optimized medium (Bahieldin et al., 2014) in further experiments.
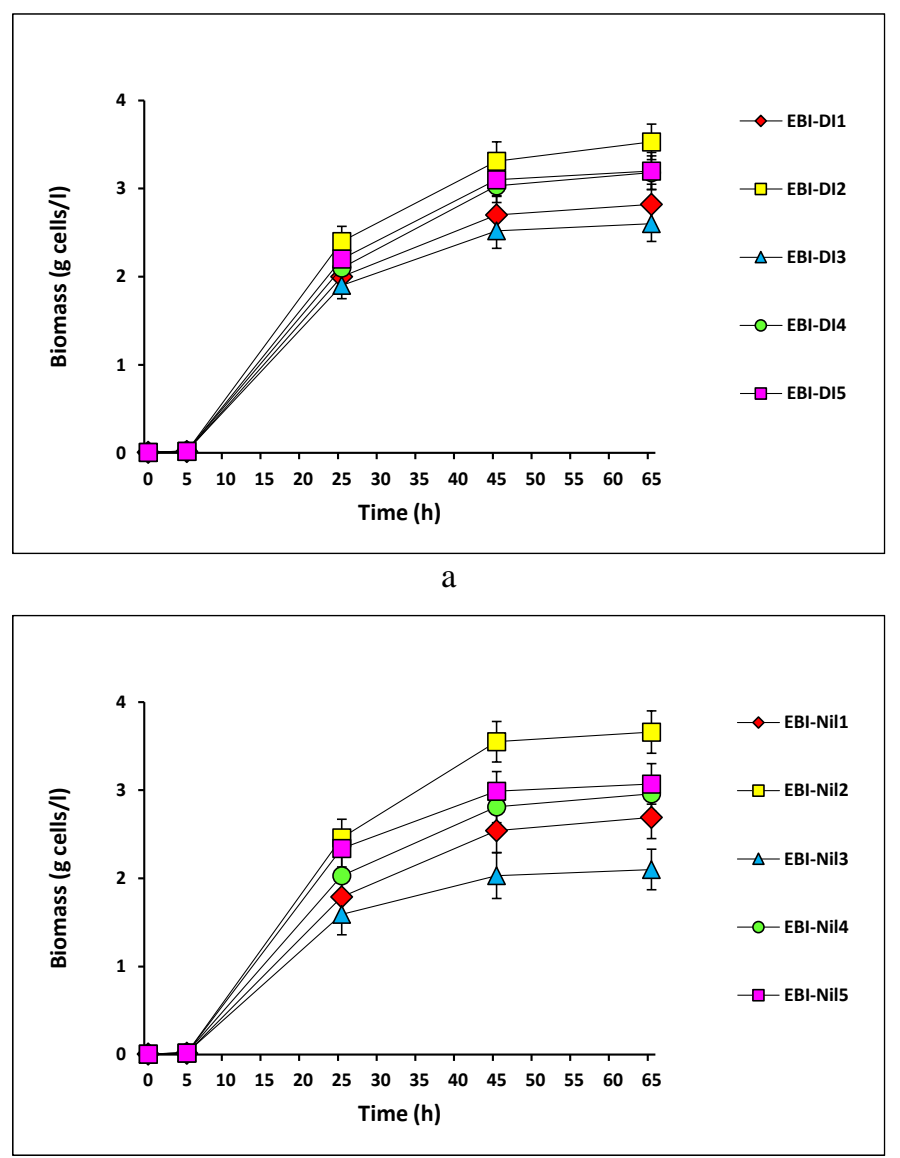

$\mathrm{b}$

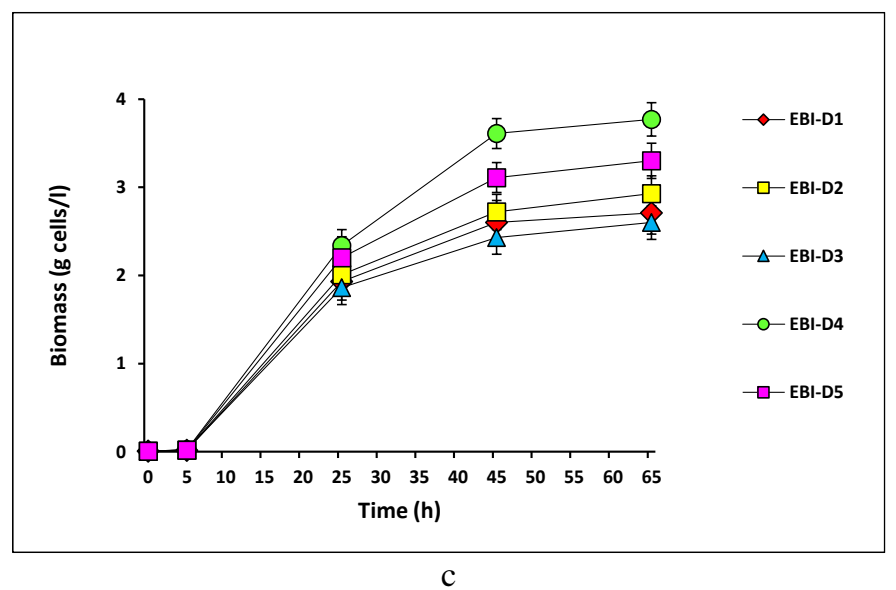

Figure 3. Growth profile of different S. cerevisiae Line 2 transformed with pTEF1/Zeo (a), pTEF1/Zeo/dxs (b) or pTEF1/Zeo/dxs/idi (c) expression vector under optimized conditions (growth in YPD medium with $0.5 \%$ glucose at $\mathrm{pH} 6.0 / 35^{\circ} \mathrm{C}$ in the presence of $200 \mu \mathrm{g} / \mathrm{ml}$ zeocin). Bars refer to values of standard error. Lines were marked via excel 


\section{Expression of dxs and idi genes in transformed S. cerevisiae lines}

Lines EBI-D4 and EBI-DI2, grown for $65 \mathrm{~h}$ at optimized conditions with galactose at $2 \%$, were subjected to molecular analysis to detect the expression of the $d x s$ and $i d i$ genes. Line 2 transformed with pTEF1/Zeo served as a control. The RT-PCR analysis (Fig. 4) revealed that the two genes were transcribed, where appropriate, and amplicons were recovered at the expected sizes. The RT-PCR analysis of transcripts from control cell resulted in the recovery of no amplicons for $d x$ s gene (Fig. 4, lane 4). Amplicon intensities of $i d i$ gene were much higher for the two Lines EBI-D4 and EBI-DI2 as compared to the control yeast cell (Fig. 4, lanes 4-6, respectively), which indicates the low expression level of the endogenous idi gene in the control (Fig. 4, lane 6) as compared to that in the two Lines EBI-D4 and EBI-DI2 (Fig. 4, lanes 4 and 5, respectively) where the gene is driven by GAL10 promoter.

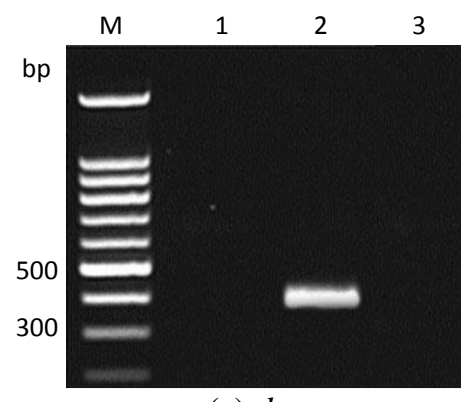

(a) $d x s$

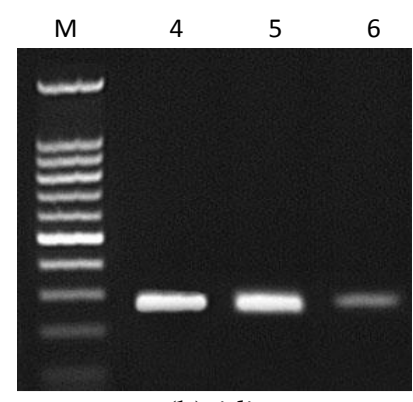

(b) $i d i$

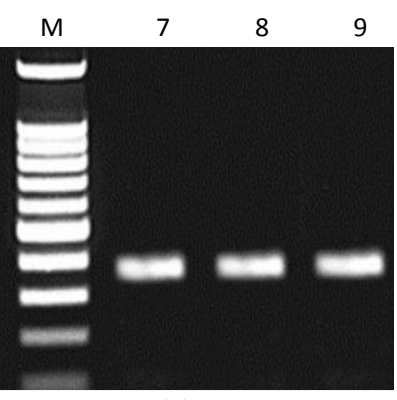

(c) actin

Figure 4. Analysis by RT-PCR of dxs and idi transcripts in EBI-D4 (lanes 1 and 4, respectively) and EBI-DI2 (lanes 2 and 5, respectively) transformed Lines of S. cerevisiae. Control cell of $S$. cerevisiae Line 2 (lanes 3 and 6, respectively) was transformed with the pTEF1/Zeo. Primers used for PCR (Table 1) were specific to dxs gene (a) to generate amplicons of $294 \mathrm{bp}$; idi gene (b) to generate amplicons of $288 \mathrm{bp}$; and actin gene (c) to generate amplicons of $337 \mathrm{bp}$. Primers specific to actin gene were used to demonstrate the transcription activity in EBI-D4 (lane 7) and EBI-DI2 (lane 8) transformed Lines of S. cerevisiae as well as S. cerevisiae Line 2 (lane 9) transformed with the pTEF1/Zeo. M refers to the 100-bp ladder (Bioron, Ludwigshafen, Germany)

\section{Synthesis of lycopene and Acetyl-CoA in S. cerevisiae transformed with dxs or dxs/idi genes}

Lycopene and Acetyl-CoA were examined for the two transformed Lines EBI-D4 and EBI-DI2 as well as for the transformed cell Line 2 w/o pTEF1/Zeo (controls) under optimized conditions in the presence of galactose at different concentrations $(0,1,2,3$ and 4\%) and measurements were made after $65 \mathrm{~h}$ cell growth (Table 2). The results of lycopene and Acetyl-CoA levels indicated no significant differences among different $S$. cerevisiae Line 2 and pTEF1/Zeo derivatives in the absence of galactose. However, the two Lines EBI-D4 and EBI-DI2 showed much higher significant levels of lycopene in the presence of galactose. This is reasonable because the onset of GAL1 and GAL10 promoter activities necessary for the expression of $d x s$ and idi genes, respectively, depends on the presence as well as the concentration of galacose in the medium. The highest levels of lycopene were scored at $2 \%$ galactose for both lines EBI-D4 and EBI$D I 2$. The latter line with both $d x s$ and $i d i$ genes significantly synthesized higher level of lycopene $(6755 \mu \mathrm{g} / \mathrm{g}$ dry cell weight) as compared to the first line with $d x$ s gene 
$(5214 \mu \mathrm{g} / \mathrm{g}$ dry cell weight), only (Table 2$)$. These results indicate the efficacy of inducing the two genes that function upstream the $c r t E B I$ genes in the MEP pathway. The same procedure was used to synthesize lycopene in E. coli but resulted in much higher levels (e.g., $8.57 \mathrm{mg} / \mathrm{g}$ dry cell weight, see Choi et al., 2010). The results of Acetyl-CoA levels across galactose concentrations indicated no significant increase or decrease within Line 2 (e.g., Line pKS1-crtEBI) as well as within its pTEF1/Zeo derivative with the increase of galactose concentration in the medium (e.g., Line EBINil2) (Table 2). However, the levels of Acetyl-CoA within Lines EBI-D4 and EBI-DI2 significantly decreased with the increase of galactose concentration in the medium up to $2 \%$. These two lines showed significantly lower levels of Acetyl-CoA as compared to either Line 2 or EBI-Nil2. The levels of lycopene and Acetyl-CoA at $65 \mathrm{~h}$ with $2 \%$ galactose in the medium reversely related among $S$. cerevisiae Line 2 with pKS1-crtEBI and pTEF1/Zeo derivatives. This indicates that biosynthesis of lycopene and AcetylCoA compete for the use of pyruvate (see Fig. 1) in yeast.

Table 2. Means ( $\pm S E$ ) of lycopene ( $\mu$ ) (a) and Acetyl-CoA ( $\mu$ moles) (b) levels per $g$ dry cell weight (DCW) of S. cerevisiae Line 2 transformant (Line pKS1-crtEBI) and those with pTEF1/Zeo derivatives. Cells were grown for $65 \mathrm{~h}$ under optimized condition with different concentrations of galactose to induce expression of bacterial dxs gene and overexpress yeast idi genes. Measurements were performed three times and means with standard errors at $5 \%$ are presented $P$-values were estimated based on the variances among transformants with different vectors and among different galactose concentrations and the variances due to the interaction between the two factors

\begin{tabular}{|c|c|c|c|c|c|}
\hline \multicolumn{6}{|c|}{ (a) Lycopene ( $\mu \mathrm{g})$} \\
\hline \multirow{2}{*}{ Vector } & \multicolumn{5}{|c|}{ Galactose $(\%)$} \\
\hline & $\mathbf{0}$ & 1 & 2 & 3 & 4 \\
\hline Line 2 (Line pKS1-crtEBI) & $3327 \pm 23$ & $3219 \pm 41$ & $3281 \pm 42$ & $2992 \pm 38$ & $3106 \pm 46$ \\
\hline pTEF1/Zeo (Line EBI-Nil2) & $3207 \pm 31$ & $3310 \pm 37$ & $3601 \pm 38$ & $3100 \pm 27$ & $3123 \pm 28$ \\
\hline pTEF1/Zeo/dxs (Line EBI-D4) & $3311 \pm 44$ & $4302 \pm 28$ & $5214 \pm 51$ & $5030 \pm 46$ & $5100 \pm 19$ \\
\hline pTEF1/Zeo/dxs/idi (Line EBI-DI2) & $3219 \pm 39$ & $4730 \pm 32$ & $6755 \pm 23$ & $6196 \pm 76$ & $6208 \pm 42$ \\
\hline Sources of variance & F value & P-value & & & \\
\hline Transformant $(\mathrm{V})(\mathrm{df}=3)$ & 3.37 & 0.0277 & & & \\
\hline Galactose $\%(\mathrm{G})(\mathrm{df}=4)$ & 6.23 & 0.0005 & & & \\
\hline$V \times G(d f=12)$ & 2.89 & 0.0058 & & & \\
\hline
\end{tabular}

(b) Acetyl-CoA ( $\mu$ moles)

\begin{tabular}{c|c|c|c|c|c}
\hline \multirow{2}{*}{ Vector } & \multicolumn{5}{|c}{ Galactose (\%) } \\
\cline { 2 - 5 } & $\mathbf{0}$ & $\mathbf{1}$ & $\mathbf{2}$ & $\mathbf{3}$ & $\mathbf{4}$ \\
\hline Line 2 (Line pKS1-crtEBI) & $3.66 \pm 0.2$ & $3.45 \pm 0.2$ & $3.19 \pm 0.2$ & $3.62 \pm 0.3$ & $3.55 \pm 0.2$ \\
pTEF1/Zeo (Line EBI-Nil2) & $3.51 \pm 0.2$ & $3.31 \pm 0.2$ & $3.33 \pm 0.3$ & $3.26 \pm 0.2$ & $3.41 \pm 0.3$ \\
pTEF1/Zeo/dxs (Line EBI-D4) & $3.62 \pm 0.3$ & $2.80 \pm 0.2$ & $1.67 \pm 0.1$ & $1.99 \pm 0.1$ & $1.93 \pm 0.1$ \\
pTEF1/Zeo/dxs/idi (Line EBI-DI2) & $3.49 \pm 0.2$ & $2.61 \pm 0.3$ & $1.50 \pm 0.1$ & $1.91 \pm 0.1$ & $1.85 \pm 0.1$ \\
\hline Sources of variance & F value & P-value & & \\
\cline { 1 - 3 } Transformant (V) (df = 3) & 4.11 & 0.0124 & & & \\
Galactose \% (G) (df =4) & 2.92 & 0.0329 & & & \\
V x G (df = 12) & 2.37 & 0.0205 & & & \\
\hline
\end{tabular}


Biosynthesis of the two compounds were also studied across yeast growth time up to $65 \mathrm{~h}$ and results indicated similar conclusions to those reached in the last experiment, where we observed an increase in lycopene synthesis in Lines EBI-D4 and EBI-D12 (Fig. 5a, triangles and circles, respectively) as compared to those in Line 2 or pTEF1/Zeo derivative (e.g., Line EBI-Nil2) (Fig. 5a, diamonds and squares, respectively). However, there were no significant changes in biosynthesis of lycopene across Line 2 and all pTEF1/Zeo derivatives beyond $45 \mathrm{~h}$ of cell growth (Fig. 5a). These results also indicate that growth of yeast cells beyond $45 \mathrm{~h}$ time point for commercial production of lycopene is unnecessary. These results align with that of the biomass production as yeast cells have gained no significant weight beyond $45 \mathrm{~h}$ of cell growth. Biosynthesis of Acetyl-CoA was increased across time up to $25 \mathrm{~h}$ for transformants EBI-D4 and EBI-DI2 (Fig. 5b, triangles and circles, respectively), while continued to increase for Lines 2 and EBI-Nil2 up to $65 \mathrm{~h}$ (Fig. 5b, diamonds and squares, respectively). On the other hand, levels of Acetyl-CoA were almost unchanged for Lines EBI-D4 and EBI-DI2 beyond $25 \mathrm{~h}$ time point. These results confirm our previous findings that biosyntheses of lycopene and Acetyl-CoA compete for the use of pyruvate in yeast.

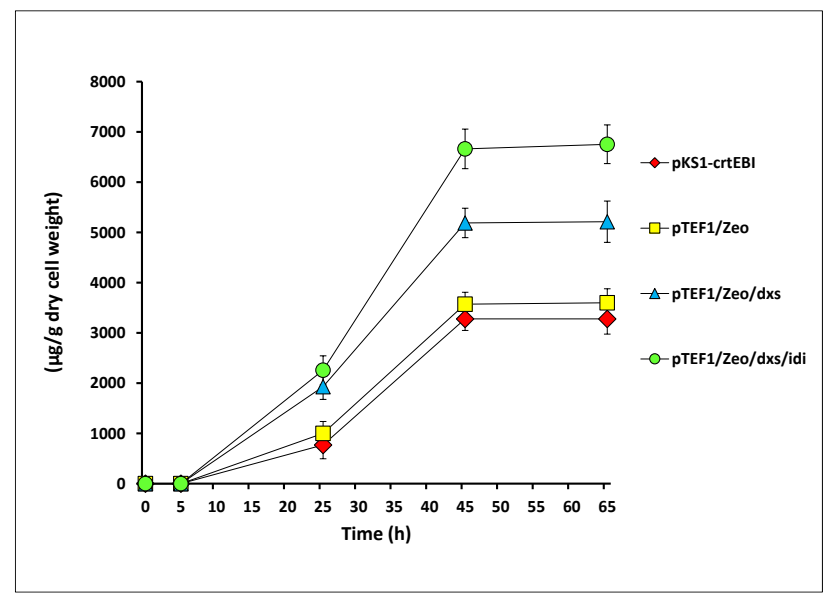

(a) Lycopene

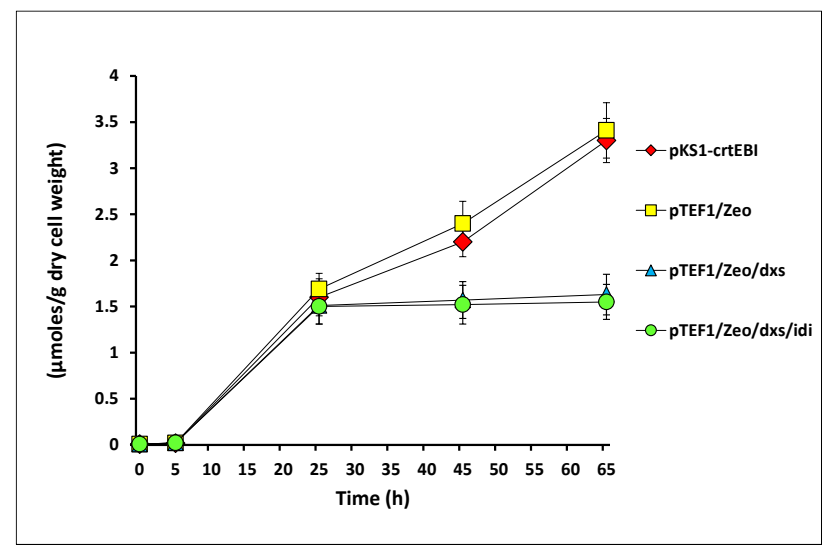

(b) Acetyl-CoA

Figure 5. Time-dependent changes across time $(0,5,25,45$ and $65 \mathrm{~h})$ in lycopene (a) and Acetyl-CoA (b) levels in EBI-D4 and EBI-DI2 transformed Lines of S. cerevisiae as well as $S$. cerevisiae Line 2 w/o the pTEF1/Zeo under optimized condition. Measurements were performed three times and means with standard errors at 5\% are presented. Bars refer to values of standard error. Lines were marked via excel 


\section{Discussion}

Promoters used for the expression of $c r t$ genes in previous studies of $C$. utilis and $S$. cerevisiae were P14 and P57, PMA, GAP, PGK (Miura et al., 1998a, b) and ADH2 (Bahieldin et al., 2014). The highest lycopene levels (1100 and $3280 \mathrm{ug} / \mathrm{g}$ dry cell weight, respectively) were reached when $\operatorname{crt} E$ gene was driven by $G A P$ promoter, $c r t B$ gene was driven by $P 14$ promoter, and $c r t I$ gene was driven by $P G K$ promoter (Miura et al., 1998a, b) or when the three $c r t$ genes were driven by $A D H 2$ promoter (Bahieldin et al., 2014). In the present study, we can claim that the use of GAL1O promoter resulted in higher expression of $i d i$ gene as well as higher levels of lycopene production. This conclusion cannot be applied for GAL1 promoter driving the $d x s$ gene as no information is available for the efficacy of utilizing this or other promoters in driving the $d x s$ gene in terms of lycopene production in yeast.

However, Lee and DaSilva (2005) also reported the superior performance of the $A D H 2$ promoter relative to the inducible CUP1 and GAL1 promoters in driving the lac $Z$ gene. We can also claim that the use of synthetic genes with codons preferred to $S$. cerevisiae cells in order to overexpress foreign genes like $d x s$ is a successful approach. These synthetic crt as well as $d x s$ genes had a high AT ratio as compared to the native genes. The results of the present work also indicate that lycopene can be produced at high levels not only by utilizing the Saccharomyces cerevisiae ADH2 promoter in driving the three crt genes but also by utilizing GAL1 and GAL1O promoters in driving the bacterial $d x s$ and the yeast $i d i$ genes, respectively. The main advantage of the $A D H 2$ promoter relative to other non-yeast promoters is the inducer-free characteristic allowing uninterrupted bioreactor operations during industrial fermentation processes (Michael Lee and DaSilva, 2005). A library of the TEF1 promoter mutants was also screened by Alper et al. (2005) and results indicated the possible generation of precise genetic control that is also a useful characteristic for industry.

Yeast (Saccharomyces cerevisiae) is considered as an attractive organism for metabolic engineering as compared to E. coli and Candida utilis as it is classified as a GRAS (generally regarded as safe) organism. The yeast cell wall does not contain any toxic components (such as pyrogens in the case of E. coli). In industry, yeast is easy to cultivate and requires no sophisticated instruments or supplies. Previous studies have indicated that $S$. cerevisiae transformed with native (Miura et al., 1998a, b) or synthesized (Bahieldin et al., 2014) crt genes accumulate lycopene. In the present study, we employed synthetic bacterial $d x s$ and overexpressed yeast idi genes. The first was constructed according to the preferable codon usage of $S$. cerevisiae, for the lycopene production. This is the first report to use $S$. cerevisiae transformed with these two genes for the successful recovery of lycopene at higher levels $(6755 \mu \mathrm{g} / \mathrm{g}$ dry cell weight) than those reported earlier. The idi gene is endogenously expressed in yeast, however, overexpression resulted in higher expression of the gene as well as higher level of lycopene production. These results prove the utility of metabolic engineering in the commercial production of lycopene.

Lycopene can be extracted from tomatoes, however, its content in the fruit is low $(\sim 0.02 \mathrm{~g} / \mathrm{kg})$, and the extraction method is difficult and relatively expensive (Sun et al., 2014). Lycopene can also be produced by chemical analysis, but this approach is complex and includes hazardous materials (Sun et al., 2014). Therefore, microbial fermentation can be considered as the method of choice (Alper and Stephanopoulos, 2008; Choi et al., 2010; Kim et al., 2011) as lycopene overproduction can take place via several strategies involving overexpression of genes required for isoprenoid synthesis to 
encode compounds of the MEP pathway (e.g., crtEBI, $d x s$ and idi). This strategy was successfully utilized in E. coli (Choi et al., 2010; Liu et al., 2020) and yeast (the present study) engineered to overproduce lycopene. Other strategies involve the knockout of (or deleting) genes functioning downstream the lycopene biosynthetic pathway (e.g., $c r t Y$ and $c r t X$ ) (Sun et al., 2014) or the increase of IPP (isopentenyl pyrophosphate) supply (Yoon et al., 2007). In addition, fermentation processes can be optimized to overproduce lycopene by adding auxiliary carbon source (Kim et al., 2011) or maintaining high $\mathrm{O}_{2}$ levels and $\mathrm{pH}$ values (Yoon et al., 2006). Most recently, Sun et al. (2014) has successfully utilized ribosome binding site libraries to modulate expression of $c r t E B I Y X, d x s$ and $i d i$ genes towards the production of substantial levels of lycopene production.

In general, we suggest that biosynthesis of lycopene and Acetyl-CoA compete for the use of pyruvate. The same conclusion was reached by Choi et al. (2010) in their study in E. coli. It is notable that the decrease in Acetyl-CoA level due to the engineering in MEP pathway is not severe as this co-enzyme is crucial for the cell in many metabolic pathways (Fatland et al., 2005). Acetyl-CoA is important for pyruvate decarboxylation, which occurs in the matrix of the mitochondria (Fig. 1), then, it enters the citric acid cycle (Choi et al., 2010). It is also an important component in the biogenic synthesis of the neurotransmitter acetylcholine and plays an essential role in regulating fatty acid synthesis and degradation (Wright et al., 2006).

\section{Conclusion}

The study aims at the assessment of crtEBI-transformed $S$. cerevisiae overexpressing two bacterial synthetic $d x s$ and yeast $i d i$ genes driven by galactose-induced promoters for lycopene production. Gene cassettes constructed in pESC-LEU were inserted in pTEF1/Zeo vector. Expression levels of the two genes were proven in crtEBItransformed $S$. cerevisiae cells resulted in highest production of lycopene $(6755 \mu \mathrm{g}$ lycopene/g dry cell weight). In contrast, the levels of Acetyl-Coenzyme A (Acetyl-CoA) was lowest in crtEBI-transformed $S$. cerevisiae cells indicating the competition for the use of pyruvate in biosynthesizing lycopene and Acetyl-CoA. We strongly recommend the use of yeast as a host for the production of lycopene utilizing metabolic engineering approaches in future trials to further improve its levels at commercial scale.

Acknowledgments. The authors acknowledge Prof. Dr. Khalid M. Al-Ghamdi, head of Department of Biological Sciences, Faculty of Science, King Abdulaziz University, Jeddah, KSA, for providing physical and logistic supports for this study.

Conflict of interests. The authors declare that they have no competing interests.

\section{REFERENCES}

[1] Alper, H., Stephanopoulos, G. (2008): Uncovering the gene knockout landscape for improved lycopene production in E. coli. - Applied Microbiology \& Biotechnology 78: 801-810.

[2] Alper, H., Fischer, C., Nevoigt, E., Stephanopoulos, G. (2005): Tuning genetic control through promoter engineering. - Proceedings of the National Academy of Sciences 102: 12678-12683. 
[3] Bahieldin, A., Gadalla, N. O., Al-Garni, S. M., et al. (2014): Efficient production of lycopene in Saccharomyces cerevisiae by expression of synthetic crt genes from a plasmid harboring the ADH2 promoter. - Plasmid 72: 18-28.

[4] Choi, H. S., Lee, S. Y., Kim, T. Y., Woo, H. M. (2010): In silico identification of gene amplification targets for improvement of lycopene production. - Applied Environmental Microbiology 76: 3097-3105.

[5] Eisenreich, W., Bacher, A., Arigoni, D., Rohdich, F. (2004): Biosynthesis of isoprenoids via the non-mevalonate pathway. - Cellular \& Molecular Life Sciences CMLS 61: 14011426.

[6] Fatland, B. L., Nikolau, B. J., Wurtele, E. S. (2005): Reverse genetic characterization of cytosolic acetyl-CoA generation by ATP-citrate lyase in Arabidopsis. - The Plant Cell 17: 182-203.

[7] Herrgård, M. J., Lee, B.-S., Portnoy, V., Palsson, B. Ø. (2006): Integrated analysis of regulatory and metabolic networks reveals novel regulatory mechanisms in Saccharomyces cerevisiae. - Genome Research 16: 627-635.

[8] Hong, J., Park, S. H., Kim, S., et al. (2019): Efficient production of lycopene in Saccharomyces cerevisiae by enzyme engineering and increasing membrane flexibility and NAPDH production. - Applied Microbiology \& Biotechnology 103: 211-223.

[9] Kim, Y.-S., Lee, J.-H., Kim, N.-H., et al. (2011): Increase of lycopene production by supplementing auxiliary carbon sources in metabolically engineered Escherichia coli. Applied Microbiology \& Biotechnology 90: 489-497.

[10] Kirschner, M. W. (2005): The meaning of systems biology. - Cell 121: 503-504.

[11] Lange, B. M., Croteau, R. (1999): Genetic engineering of essential oil production in mint. - Current Opinion in Plant Biology 2: 139-144.

[12] Lange, B. M., Wildung, M. R., McCaskill, D., Croteau, R. (1998): A family of transketolases that directs isoprenoid biosynthesis via a mevalonate-independent pathway. - Proceedings of the National Academy of Sciences 95: 2100-2104.

[13] Lange, B. M., Rujan, T., Martin, W., Croteau, R. (2000): Isoprenoid biosynthesis: the evolution of two ancient and distinct pathways across genomes. - Proceedings of the National Academy of Sciences 97: 13172-13177.

[14] Lee, P., Schmidt-Dannert, C. (2002): Metabolic engineering towards biotechnological production of carotenoids in microorganisms. - Applied Microbiology \& Biotechnology 60: 1-11.

[15] Lee, S. Y., Lee, D.-Y. and Kim, T. Y. (2005): Systems biotechnology for strain improvement. - Trends in Biotechnology 23: 349-358.

[16] Liu, N., Liu, B., Wang, G., et al. (2020): Lycopene production from glucose, fatty acid and waste cooking oil by metabolically engineered Escherichia coli. - Biochemical Engineering Journal 155: 107488.

[17] Lois, L. M., Campos, N., Putra, S. R., et al. (1998): Cloning and characterization of a gene from Escherichia coli encoding a transketolase-like enzyme that catalyzes the synthesis of D-1-deoxyxylulose 5-phosphate, a common precursor for isoprenoid, thiamin, and pyridoxol biosynthesis. - Proceedings of the National Academy of Sciences 95: 2105-2110.

[18] Michael Lee, K., DaSilva, N. A. (2005): Evaluation of the Saccharomyces cerevisiae $A D H 2$ promoter for protein synthesis. - Yeast 22: 431-440.

[19] Miura, Y., Kondo, K., Saito, T., et al. (1998a): Production of the carotenoids lycopene, $\beta$ carotene, and astaxanthin in the food yeast Candida utilis. - Applied Environmental Microbiology 64: 1226-1229.

[20] Miura, Y., Kondo, K., Shimada, H., et al. (1998b): Production of lycopene by the food yeast, Candida utilis that does not naturally synthesize carotenoid. - Biotechnology \& Bioengineering 58: 306-308.

[21] Price, N. D., Reed, J. L. and Palsson, B. Ø. (2004): Genome-scale models of microbial cells: evaluating the consequences of constraints. - Nature Reviews Microbiology 2: 886. 


$$
-5292-
$$

[22] Rodriguez-Concepción, M., Boronat, A. (2002): Elucidation of the methylerythritol phosphate pathway for isoprenoid biosynthesis in bacteria and plastids. A metabolic milestone achieved through genomics. - Plant Physiology 130: 1079-1089.

[23] Römer, S., Fraser, P. D., Kiano, J. W., et al. (2000): Elevation of the provitamin A content of transgenic tomato plants. - Nature Biotechnology 18: 666.

[24] Sevgili, A., Erkman, O. (2019): Improved lycopene production from different substrates by mated fermentation of Blakeslea trispora. - Foods 8: 120.

[25] Sun, T., Miao, L., Li, Q., et al. (2014): Production of lycopene by metabolicallyengineered Escherichia coli. - Biotechnology Letters 36: 1515-1522.

[26] Wright, T. C., Cant, J. P., Brenna, J. T., McBride, B. W. (2006): Acetyl CoA carboxylase shares control of fatty acid synthesis with fatty acid synthase in bovine mammary homogenate. - Journal of Dairy Science 89: 2552-2558.

[27] Yoon, S. H., Lee, Y. M., Kim, J. E., et al. (2006): Enhanced lycopene production in Escherichia coli engineered to synthesize isopentenyl diphosphate and dimethylallyl diphosphate from mevalonate. - Biotechnology \& Bioengineering 94: 1025-1032.

[28] Yoon, S.-H., Kim, J.-E., Lee, S.-H., et al. (2007): Engineering the lycopene synthetic pathway in E. coli by comparison of the carotenoid genes of Pantoea agglomerans and Pantoea ananatis. - Applied Microbiology \& Biotechnology 74: 131-139. 